\title{
Ueber Phenolverbindungen;
}

\section{von Demselben.}

Die Beobachtung, dafs sicb Phenol leicht mit neutralem Chininsulfat verbinde, welche im vergangenen Jahre von $J u l$. Jobst und mir gemacht und inzwischen mitgetheilt *) wurde, bestimmte mich, auch die anderen Chinaalkaloïde in dieser Richtung zu prüfen. Zwar ist diese Untersuchung bei weitem noch nicht zum Abschlufs gelangt; allein der Umstand, dafs Cotton den gleichen Gegenstand zu seinen Untersuchungen gewählt hat, verunlafst mich, das Folgende jetzt schon mitzutheilen.

Vor allem dürften die betreffenden Cinchonidinverbindungen interessiren. Bringt man nämlich zur heifsen Auflösung von neutralem Cinchonidinsulfat in Wasser Phenolwasser $(1: 25)$, so krystailisirt beim Erkalten derselben phenolschwefelsaures Cinchonidin, das durch Unkrystallssiren aus kochendem Wasser gereinigt werden kann. Es bildet dann weifse glänzende geruchlose Prismen, die entsprechend den folgenden Bestimmungen nach der Formel $2 \mathrm{C}_{\mathbf{8} v} \mathrm{H}_{\mathbf{2}} \mathrm{N}_{\mathbf{8}} \mathrm{O}$, $\mathrm{SO}_{9} \cdot \mathrm{C}_{6} \mathrm{H}_{6} \mathrm{O}+5 \mathrm{H}_{2} \mathrm{O}$ zusaminengesetzt sind :

I. 0,4325 Grm. lufttrockene Substans gaben $0,0476 \quad \mathrm{H}_{8} \mathrm{O}$ bei $110^{\circ}$ und $0,115 \mathrm{SO}_{4} \mathrm{Ba}$.

II. $0,40 \bar{j}$ Grm. Iufttrockene Substanz gaben $0,0446 \mathrm{H}_{2} \mathrm{O}$ bei $110^{\circ}$.

1I. 0,3995 Grm. luftrockene Bubstanz, zweimal aus Wasser umkrystallisirt, gaben bei $110^{\circ} 0,0425 \mathrm{H}_{\mathrm{z}} \mathrm{O}$.

\begin{tabular}{lcccc} 
& & \multicolumn{3}{c}{ Gefunden } \\
\cline { 3 - 6 } & Berechnet & L & II. & III. \\
SO & 9,09 & 9,12 & - & - \\
$\mathbf{H}_{2} \mathrm{O}$ & 10,23 & 10,98 & 10,98 & $10,64$.
\end{tabular}

*) Diese Annalen $180,248$.

**) Bull. soc. chim. D4, 535 . 
Das phenolschwefelsaure Cinchonidin lōst sich bei $15^{\circ}$ in 425 Theilen Wasser, leicht in kochendem Wasser. Aus seiner kalt gesättigten wässerigen Lósung wird es durch Phenolwasser zum gröfseren Theil ausgefällt. Heifser Alkohol löst es leicht und scheidet beim Erkalten einen Theil des Salzes in hübschen Prismen ab.

Das Salz färbt sich mit Eisenchlorid schwach dunkelgelb. Wenn somit die für Phenol charakteristische Reaction mit Eisenoxyd ausbleibt und aus diesem Grunde auf die Abwesenheit von Phenol [bezw. von Phenolhydroxyl *)] in dieser Verbindung geschlossen werden müfste, so scheiden doch anderenseits verdünnte Säuren daraus Phenol sehr leicht ab, welches durch Ausschütteln mit Aether oder Petrolbenzin gewonnen werden kann. Auch durch Basen, wie z. B. durch Ammoniak, wird Phenol abgeschieden, während gleichzeitig das Alkaloĩd ausfällt. Die Carbolsäure hat in dieser Verbindung ihre ätzende Wirkung eingebüfst.

Auch mit Cinchonidinchlorhydrat bildet Phenol eine woht charakterisirte Verbindung. $Z u$ ihrer Darstellung löst man gleiche Molecule von Cinchonidinsalz und Phenol in heifsem Wasser, worauf beim Erkalten der Lösung die neue Verbindung krystallisirt, welche man zur völligen Reinigung zweckmäfsig aus kochendem Wasser umkrystallisirt.

Diese Verbindung, weifse körnige Krystalle bildend, verliert im unbedeckten Tiegel auf $100^{\circ}$ erhitzt aufser Krystallwasser erhebliche Mengen von Phenol. Schon nach kurzer Zeit beträgt der Gewichtsverlust 10 bis 12 pC. Derselbe steigert sich dann allmälig, bis er schliefslich bei $120^{\circ}$ etwa 22 pC. beträgt. Während dem (bei $120^{\circ}$ ) nimmt der Rückstand ein braunes Aussehen an und löst sich dann in Wasser mit brauner Farbe.

-) Dieso Annalen 158, 158. 
Wird aber das Salz in leicht verschlossenem Probirrohr auf etwa $100^{\circ}$ erhitzt, Bo schmilzt es zuerst und verliert fast nur Wasser. Die hierauf erkaltete Masse bedeckt sich mit einer krystallinischen Kruste, bleibt jedoch im Innern während langer Zeit flüssig. Diese flüssige Masse stellt offenbar die wasserfreie Verbindung vor.

Auch wenn die krystallisirte Substanz mit heirsem Wasser behandelt wird, so schmilat anfanglich ein Theil derselben.

Das phenolchlorwasserstoffsaure Cinchonidin oder wohl richtiger chlorwasserstoffaaures Phenolcinchonidin genannt, lōst sich leicht in Alkohol und in heifsem Wasser, wăhrend 1 Theil Salz bei $15^{\circ} 46$ Theile Wasser zur Lösung erfordert. Aus letzterer Lösung wird es durch Phenolwasser zum Theil wieder abgeschieden. In Chloroform löst sich das Salz leicht, sehr wenig dagegen in Aether.

Es zeigt in seiner Auflösung in 97 vpc. Alkohol bei $t=15^{\circ}$ und $p=2$ ein Drehungsvermögen ron $(\alpha)_{D}=$ $-83,75^{\circ}$, woraus sich für das Alkaloïd $(\alpha)_{\mathfrak{D}}=-124,12^{\circ}$ berechnet, nach den folgenden Ermittelungen.

Das im Exsiccator getrocknete Salz ergab nàmlich für :

I. $0,569 \mathrm{Grm}$. Substanz $0,1785 \mathrm{AgCl}$.

II. $0,716 \mathrm{Grm}$. Substanz $0,2246 \mathrm{AgCl}$.

Mit Rücksicht auf den qualitativen Befund läfst sich für dieses Salz nur die Formel $\mathrm{C}_{80} \mathrm{H}_{84} \mathrm{~N}_{8} \mathrm{O}, \mathrm{C}_{6} \mathrm{H}_{6} \mathrm{O}, \mathrm{HCl}+\mathrm{H}_{2} \mathrm{O}$ aufstellen, welche in 100 Theilen

\begin{tabular}{|c|c|c|}
\hline & & \\
\hline verlangt & I. & II. \\
\hline 7,77 & 7,76 & $7,74$. \\
\hline
\end{tabular}

Zu Eisenchlorid verhält sich diese Verbindung wie die vorige, auch hat in ihr das Phenol seine ätzenden Eigenschaften verloren, doch kann dasselbe durch Säuren leicht wieder regenerirt werden.

Um Aufklärung über die Constitation dieser Verbindung zu erhalten, wurden zwei weitere Versuche ausgeführt. 
1) Es wurde chlorwasserstoffsaures Phenolcinchonidin in Phenolwasser gelöst und aus der verdünnten Lösung bei gewöhnlicher Temperatur mit Platinsolution ausgefällt, in der Absicht, auf solche Weise ein chlorwasserstoffsaures Phenolcinchonidinplatinchlorid darzustellen. Das in diesem Versuche resultirende Salz war indefs nur chlorwasserstoffsaures Cinchonidinplatinchlorid.

2) Cinchonidin wurde mit Phenol zu gleichen Moleculen in heifsem verdünntem Alkohol gelöst behufs der Darstellung von Phenolcinchonidin. Beim Erkalten der Lösung schied sich zunächst eine ölige Flüssigkeit ab, die sich nach einiger Zeit in hübsche farblose glasglänzende Prismen uınsetzte. Dieselben erwiesen sich in der That als eine Verbindung von Cinchonidin Init Phenol. Dieses Phenolcinchonidin verhielt sich zu Eisenchlorid wit das obige Chlorhydrat; verdūnnte Salpetersäure schied aus ihm Phenol ab *).

Hiernach unterliegt es wohl keinem Zweifel, dafs die Anlagerungen von Phenol in den obigen Verbindungen sowohl wie in den früher erwähnten Chininsalzen an die betreffenden Alkaloîde und nicht an die entsprechenden Saize erfolgten. Indefs würde diese Anschauungsweise zwei Reihen von Verbindungen voraussetzen, nämlich von Verbindungen von gleicher Anzahl von Moleculen der Componenten, und Verbindungen von einem Molecul Phenol mit zwei Moleculen Alkaloïd. Darüber können ineines Erachtens nur Versuche ontscheiden; so lange dieses nicht geschehen ist, sollte man obige Verbindungen von Phenol und Salzen in derselben Weise bezeichnen, wie es früher von Jul. Jobst und mir schon geschehen ist.

Uebrigens wird nicht in Abrede gestellt werden können, dafs auch eine Verbindung von Phenol mit Chinin existirt,

*) Weitures über diese Verbindung erfolgt spater. 
obwohl alle Versuche, diese Verbindung in einer greifbaren charakterisirten Form zu erhalten, bislang vergeblich waren. Weitere Versuche mit Cinchonin, Conchinin und Chinamin werden lehren, ob sich solche Verbindungen mit diesen Basen darstellen lassen. Bislang wurden nur Salze dieser Busen auf das genannte Verhalten geprüft.

Es ergab sich, dafs sich die Conchinin- und Cinchoninsalze wesentlich verschieden verhalten von den entsprechenden Cinchonidinsalzen. Zwar gehen diese Sulze mit Phenol Verbindungen ein, allein diese sind amorph, ojlig, in kaltem Wasser leicht löslich. Ein Ueberschufs von Phenolwasser scheidel sie aus wässeriger Lösung ab.

In der gleichen Weise wie letztere Salze verhalten sich Chinicin- und Cinchonicinsulfat. Beide werden zwar aus ihrer wässerigen Lösung durch Phenolwasser gefallt, allein diese Fällung ist sehr unvollständig.

Gereinigtes Chinö̈dinsulfat bzw. sogenanntes Chinin. sulfur. amorphum giebt erst bei grofsem Ueberschufs von Phenolwasser eine bleibende amorphe Fällung. Doch trübt sich diese Lösung schon beim Zufügen von geringen Mengen Phenolwasser, ohne dars eigentlich eine Fällung eintritt.

Chinaminsulfat giebt auffullender Weise keine Fällung mit Phenolwasser. Demnach zeigen die neutralen Sulfate der bis jetzt näher bekannten Chinaalkaloïde wesentliche $V_{t e r-}$ schiedenheiten in ihrem Verbalten zu Phenolwasser, so dafs letzteres auf diesem Gebiete als ein nicht ganz unwillkommenes Reagens betrachtet werden darf.

Zwar wurde schon früher von $\mathrm{Hager}$ *) das Phenolwasser als Reagens auf Chinaalkaloïde, nämlich zur Entdeckung von Verfälschung der Chinarinden mit Chinoïdin verwendet, jedoch damals der Gegenstand nicht ganz klar gestellt.

*) Dessen Commertar zur doutschen Reichspharmacopöe, 530. 
Denn nach dem Verhalten des gereinigten Chinoidinsulfats zu Phenolwasser zu urtheilen, müfste die Menge von Chinoïdin oder Chinoüdinsalz schon sehr beträchtlich sein, bis die fragliche Reaction sicher eintritt. Indefs wäre es denkbar, dafs Hager seine Controlversuche mit einem Chinoïdin ausführte, weiches viel Cinchonidin enthielt, für welchen Fall die Reaction schon bei einem geringeren Grad der Verfälschung eintreten würde.

Oder ist die Empfindichkeit dieser Reaction von einem besonderen, bis jetzt noch unbekannten Alkaloïd abhängig? Diese Frage habe ich mir schon längst gestellt, bin aber leider noch nicht in der Lage, eine bestimmte Antwort hierauf zu geben.

Schliefslich möchte ich darauf hinweisen, dafs während das Phenol ein vorzügliches Mittel zur Beurtheilung der Qualität der Chinaalkaloïde ist, letztero umgekehrt als Reagentien auf Phenol dienen können. Ich bin sogar der Meinung, dars einige Salze der Chinaalkaloïde ähnliche Verwendung finden können, wie gewisse Bisulfite zur Erkennung und Abscheidung von Aldehyden und Acetonen. Insbesondere wird sich hierzu das Cinchonidinsulfat oder das käufliche, durch Seignellesalz fällbare Chinidinsulfat eignen.

\section{Ueber Aricin und verwandte Substanzen; von Demselben.}

Die Existenz des Aricins wurde früher *) von mir deshalb in Frage gestellt, weil alle meine Versuche, welche die

-) Diese Annalon I68, 263. 